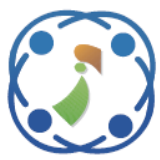

\title{
Eagle Strategy with Cauchy Mutation Particle Swarm Optimization for Energy Management in Cloud Computing
}

\author{
Archana Kollu $^{1 *} \quad$ Sucharita Vadlamudi $^{2}$ \\ ${ }^{1,2}$ Department of Computer Science and Engineering, Koneru Lakshmaiah Education Foundation, \\ Guntur District Andhra Pradesh, India \\ * Corresponding author’s Email: sai.archna@gmail.com
}

\begin{abstract}
Energy management of the cloud datacentre is a challenging task, especially when the cloud server receives a number of the user's request simultaneously. This requires an efficient method to optimally allocate the resources to the users. Resource allocation in cloud data centers need to be done in optimized manner for conserving energy keeping in view of Service Level Agreement (SLA). We propose, Eagle Strategy (ES) based Modified Particle Swarm Optimization (ES-MPSO) to minimize the energy consumption and SLA violation. The Eagle Strategy method is applied due to its efficient local optimization technique. The Cauchy Mutation method which schedules the task effectively and minimize energy consumption, is applied to the proposed ES-MPSO method for improving the convergence performance. The simulation result shows that the energy consumption of ES-MPSO is 42J and Particle Swarm Optimization (PSO) is 51J. The proposed method ES-MPSO achieves higher efficiency compared to the PSO method in terms of energy management and SLA.
\end{abstract}

Keywords: Cauchy mutation, Convergence, Eagle strategy, Particle swarm optimization, Energy management, Service level agreement.

\section{Introduction}

Cloud computing is developed as one of the most popular technologies that provide on-demand services to the users. Cloud computing is used by many users due to its scalability and flexibility [1]. Cloud services offer a pool of resources like CPU, memory, software services and these services are provided to the user based on the pay-per-use model. In addition, cloud based service providers' delivers on-demand content that leads to many challenges for organizations [2]. Massive deployment of cloud datacenter comes with the cost of more energy consumption and excessive carbon footprint. This requires the green initiative in the datacenters for efficient energy consumption strategy [3]. Allocation of the task in the different Virtual Machine (VM) helps to reduce the energy consumption and effective resource utilization in the cloud datacentre. However, proper utilization of resources and efficient energy consumption in cloud datacenter are the main challenges of cloud datacenter [4]. One of the major challenges of the cloud service provider is to satisfy the demand of the user and manage the energy consumption efficiently [5].

An efficient load balancing strategy is necessary to improve utilization of the cloud resource and increase the performance of the cloud computing. This is important to find the best solution for the cloud datacenter to maximize the efficiency of a system or minimize the processing cost. Hybridization of metaheuristic optimization method can improve the convergence speed and quality of solutions [6, 7]. Some of the important factors to consider for resource allocation are energy management, makespan, memory usage and violation of SLA, etc. Each VM requires the required amount of CPU, memory, storage and bandwidth to satisfy the needs of users [8-10]. In the past few years, the researchers have designed various intelligence computation methods like differential evolution, Ant colony, PSO, Artificial bee colony (ABC), etc. to 
minimize the energy consumption and SLA violations. But, most of them are insufficient to achieve good results in all aspects (i.e. the existing algorithms are easily trapped in local optima). In addition, hybrid meta-heuristic algorithms have been designed to achieve less energy consumption, but it has more makespan compared to other techniques [11]. In this work, Eagle Strategy based Modified Particle Swarm Optimization (ES-MPSO) method is proposed for the efficient energy management of the cloud datacenter. In order to avoid trap of local optimum of PSO, the convergence performance is improved by introducing the Cauchy mutation method in PSO as MPSO. In addition, the effectiveness of the local optimization technique is improved by eagle strategy. The developed optimization method is simulated in cloud environment and compared with the existing methods.

The experimental result shows that the proposed ES-MPSO method outperforms the existing PSO method in terms of energy management, makespan and SLA violation.

The paper is organized as follows, the literature review on energy management of cloud datacenter is presented in the Section 2, the Eagle strategy and modified PSO is discussed in the Section 3, Experimental design of the method is presented in the Section 4, the experimental result of the proposed method is presented in the Section 5 and the conclusion is drawn in Section 6.

\section{Literature review}

Cloud datacenter consumes more energy to store and process large amount of user data. Various methods have been proposed for optimizing the energy management in cloud datacenter. Latest researches on optimization of energy management in cloud datacenters is reviewed in this section.

Zhou, Abawajy, Chowdhury, Hu, Li, Cheng, Alelaiwi and $\mathrm{Li}$ [12] proposed two adaptive energy aware methods to improve the energy efficiency and minimize SLA violation in the cloud. This method considered the CPU and memory resources in the allocation of VM. The experimental result showed that developed method achieved lower energy consumption and maintained low SLA violation. Even though, the method consumes less energy and SLA violations, but it increases the operation cost.

Malekloo, Kara and El Barachi [13] developed Multi-Objective Ant Colony Optimization (MACO) to minimize the SLA violations and energy consumption, which also improved the QoS for the VM allocation. The multi-objective consolidation method optimized the resource allocation based on the VM migration. The MACO method was tested through the simulation of small to medium datacentre settings and compared with the other metaheuristics method. The parameters CPU, memory, storage and network bandwidth were considered for resource management. The simulation result indicated that the Multi-Objective Ant Colony Optimization (MACO) method achieved higher performance in maintaining the energy efficiency and QoS. However, if the number of objective functions increase then it results in high costs in terms of time complexity and a degradation in performance with respect to some of the metrics, due to the difficulty of finding solutions that satisfy all those requirements, simultaneously.

Prassanna and Venkataraman [14] developed Nature-inspired Meta-heuristic Threshold based Firefly Optimized Lottery Scheduling (NMT-FOLS) for the VM consolidation. The adaptive regression method was used for the prediction of the workload state. The multi-objective firefly algorithm was implemented for the task scheduling in the normal state and in the bursty time, multi-objective firefly lottery scheduling algorithm was used. The optimization method scheduled the user task for the optimal VM to improve the resource allocation. The two existing techniques two-stage strategy and Resource-Aware Load Balancing Algorithm (RALBA) were used to test the efficiency of the NMT-FOLS method. The experimental result showed that the firefly based method achieved higher efficiency in the scheduling and reduced the SLA violation of the cloud. However, if the number of user requested tasks increase then the NMT-FOLS reduces the prediction accuracy and increase memory usage.

Jena and Mohanty [15] presented Genetic algorithm based task scheduling algorithm for the VM migration. The method was divided into two phase Genetic algorithm based task scheduling and shortest task first scheduling. The method involves mapping the tasks to VM to minimize makespan time and to maximise customer satisfaction. The method was tested in the simulation environment and compared with the existing method. The simulation result showed that developed Genetic algorithm based method achieved higher performance compared to existing technique. Even though, the method achieved good performance, but the energy consumption, latency arbitration, running cost and data locality cost were not considered.

$\mathrm{Li}$, Dong, Zuo and $\mathrm{Wu}$ [16] proposed host overloading or underloading detection method and linear regression prediction model for SLA-aware and energy efficient VM consolidation. Eight methods were used to calculate the error of the model. 
The developed method was evaluated in the simulation environment to analyse the performance. The result showed that the developed method minimizes the energy consumption and reduced SLA violation in the cloud. An adaptive lower utilization threshold was applied based on the Interquartile Range (IR). However, the energy consumption of the datacentre and number of shutdown hosts increase due to high reactivation of hosts.

Gamal, Rizk, Mahdi and Elnaghi [17] combined the osmotic behaviour with hybrid metaheuristics and formed an algorithm called osmotic hybrid artificial bee and ant colony (OH_BAC) for effective load balancing. The objective of the OH_BAC method was to address the issues of traditional bio-inspired algorithms for balancing the loads between VMs. The simulations were conducted to test the effectiveness of OH_BAC with Hybrid artificial Bee and Ant Colony optimization (H_BAC), Artificial Bee Colony (ABC) and Ant colony Optimization (ACO) in terms of energy consumption, SLA violations, number of shutdown hosts and number of VM migrations. Though, the OH_BAC method achieved better performance, its SLA Violations Time per Active Host (SLATAH) is higher than other algorithms.

From the analysis of existing techniques, it is understood that energy consumption is high due to reactivation of the hosts and increases the memory usage. The Cauchy mutation process is added with the PSO to improve the convergence performance that minimizes the energy consumption and SLA violations. The inertia weight based on Cauchy mutation is added in the PSO to escape from the locally trap in the search process.

\section{Proposed method}

Optimal allocation of VM is a challenging task because of a number of users' requests to the cloud datacenter simultaneously. Though many existing methods have shown considerable performance in the VM allocation to improve the efficiency, but the SLA violation of these methods is high.

The objective of the proposed method is to reduce the consumption of energy and SLA violations for cloud providers under heterogeneous cloud environment. The formula for energy consumption is defined in the following sub-section.

\subsection{Definition of energy consumption}

In cloud datacenters the network interfaces, CPU, disk storage and memory are used to compute the energy consumption. In this work, the energy consumption, which is caused by the CPU is studied.
The linear model is the one of the most common energy consumption models, where there is a linear correlation between CPU utilization and energy consumption. Therefore, the definition of the energy consumption (EC) of the $i^{t h}$ server at time $t$ in the proposed method is presented in the Eq. (1).

$=\left\{\begin{array}{cc}E C_{i}(t) & \text { if } H_{i} \text { is idle } \\ E C_{i}^{\text {idle }}+(1-\rho) \times E C_{i}^{\text {full }} & \text { if } H_{i} \text { is busy }\end{array}\right.$

If the server or $H_{i}$ is idle, the energy ratio of the idle processor (i.e. $70 \%$ ), which is represented by the static coefficient called $\rho$. Under full server load, the energy consumption of the physical node $i$ is defined as $E C_{i}^{f u l l}$. At time t, the CPU utilization dynamically changes according to the workload as an independent variable that is denoted as $U(t)$. The Eq. (2) defines the energy consumption of server $H_{i}$ throughout the process as follows:

$$
E C_{i}=\int_{t_{0}}^{t_{1}} E C_{i}(t) d t
$$

Then, the mathematical Eq. (3) is used to compute the total energy consumption of a cloud datacentre with $\mathrm{n}$ nodes as follows.

$$
E C=\sum_{i=1}^{n} x_{i} E C_{i}
$$

$$
\text { Where, } x_{i}=\left\{\begin{array}{cc}
0 & \text { if the } H_{i} \text { is shutdown } \\
1 & \text { other }
\end{array}\right.
$$

\subsection{Definitions of SLA violation metrics}

In the cloud datacentre, QoS is defined by the SLA, where users obtain the services by cloud providers. An SLA violation occurs when users place excessive demands on the datacenter. If the host is resigned, its computing performance is significantly reduced. Similarly, additional resources are consumed by the VM migration process. The degree of SLA violation directly reflects the availability and reliability of the system. In this work, different metrics are selected to measure the degree of SLA violations.

i. SLATPAH: Each active host violated the SLA at a percentage time is expressed in Eq. (4).

$$
S L A T P A H=\frac{1}{n} \sum_{i=1}^{n} \frac{T_{i}^{f}}{T_{i}^{a}}
$$

Where, the number of servers is described as $n$; 
Total time that host $i$ as $T_{i}^{a}$, which is active; the total time that host $i$ experiences full load as $T_{i}^{f}$.

ii. SLAPDM: Migration causes the performance degradation that is expressed as Eq. (5).

$$
\left.S L A P D M=\frac{1}{m} \sum_{j=1}^{m} \frac{C_{j}^{d}}{C_{j}^{r}}=\frac{1}{m} \sum_{j=1}^{m} \frac{0.1 \times C_{j}}{C_{j}^{r}}\right)
$$

Where, the number of VMs in the datacenter is illustrated as $m$; total required CPU capacity (in MIPS) during the life cycle of $\mathrm{VM} j$ as $C_{j}^{r}$; a decrease in performance due to $\mathrm{VM} j$ migration as $C_{j}^{d}$. It is generally believed that the $10 \%$ of the CPU computing power is minimized by VM migration. The $C_{j}$ is defined as CPU power of VM $j$. Therefore, the product of (4) and (5) defines the overall service level agreement violation (SLAV) as in Eq. (6), i.e.

$$
S L A V=S L A P A H \times S L A P D M
$$

Therefore, these two problems must be minimized by using the proposed method, which is discussed in the below section.

In this work, Eagle Strategy with Modified Particle Swarm Optimization (ES-MPSO) method is proposed to improve the efficiency and reduce the SLA violation in the cloud datacenter. The Eagle Strategy is capable of intensive local search with the effective local optimizer method. This helps to improve the searching method of the proposed system. The Cauchy Mutation method is applied in the PSO that improve its convergence performance. The developed method is simulated in the CloudSim and compared with the existing optimization methods. The flow diagram of the proposed ES-MPSO method is presented in Fig. 1.

\subsection{Eagle strategy}

Eagle strategy is inspired by the foraging behaviour of eagles that fly random in analogy to the Levy flights [18]. Various optimization algorithms have been used by ES method to process local search for scheduling the tasks. This method is similar to the random restart hill climbing method, but there are two important differences. The Eagle Strategy is a two-stage method global search randomization and an intensive local search. The Eagle Strategy method uses the Levy walks to explore the global search space more effectively.

Eagle strategy makes the global search in the $n$ dimensional space based on Levy flights. An intensive local optimizer Particle Swarm Optimization (PSO) is used for local search to find

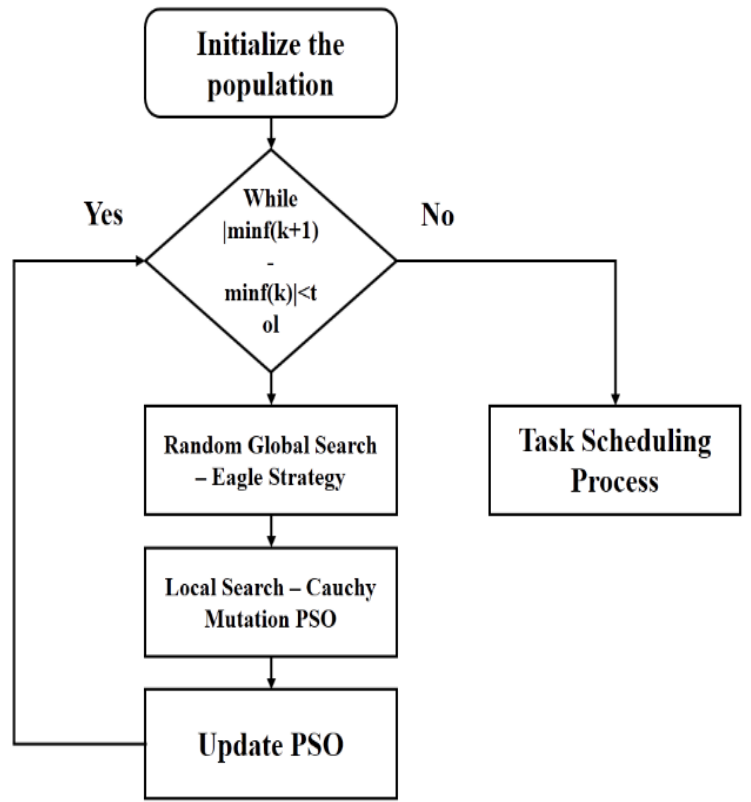

Figure. 1 The flow diagram of proposed ES-MPSO VM allocation

probable solution. This PSO method is capable of local search. The searching process is repeated with new global search in the different area.

In order to achieve better results, the ES method considered the benefits of different two algorithms $\mathrm{ACO}$ and $\mathrm{ABC}$, which is presented as case study in the experimental section. Levy distribution [18] is presented in Eq. (7).

$$
\begin{gathered}
L(s) \sim \frac{\lambda \Gamma(\lambda) \sin \left(\frac{\pi \lambda}{2}\right)}{\pi} \frac{1}{S^{1+\lambda}}, \\
\left(s \gg s_{0}>0\right),(1<\lambda \leq 3)
\end{gathered}
$$

Where the standard gamma function is denoted as $\Gamma(\lambda)$ and the step length is set as $s$. If $\lambda=3$, then it becomes special case. But, in this case, $\lambda=2$ is used, so the Levy walks become the Cauchy distribution.

\subsection{Particle swarm optimization}

The PSO uses parameter particle number, accelerate constant, inertia weight and maximum limited velocity and starts the optimization process based on the Initial Population (Particles). Each particle has a possible solution for the main problem and here, the local search problem is processed as a part of $n$-dimensional space. Each particle's position is identified by $x_{i}^{k}=\left(x_{i 1}^{k}, x_{i 2}^{k}, \ldots, x_{i n}^{k}\right)$ and velocity is identified by $V_{i}^{k}=\left(V_{i 1}^{k}, V_{i 2}^{k}, \ldots, V_{i n}^{k}\right)$ in the variable space. Velocity and position are updated based on the Eq. (8) and (9), respectively. If the particles have the best solution, then it is sent to the next process. The best position is denoted as the pbest and best velocity is denoted as gbest. 


$$
\begin{aligned}
v_{i}^{k+1}= & w_{i} v_{i}^{k}+c_{1} r_{1}\left(\text { pbest }_{i}-x_{i}^{k}\right)+ \\
& c_{2} r_{2}\left(\text { gbest }_{i}-x_{i}^{k}\right) \\
x_{i}^{k+1}= & x_{i}^{k}+v_{i}^{k+1}
\end{aligned}
$$

The weight function is presented in Eq. (10).

$$
w_{i}=w_{\max }-\frac{w_{\max }-w_{\min }}{k_{\max }} \times k
$$

Where $r_{1}$ and $r_{2}$ are the random numbers selected in the range of $[0,1]$ and $c_{1}, c_{2}$ are acceleration coefficients that analyze particle motion.

The Cauchy distribution is developed based on the continuous probability distribution [19] and the mathematical expression of this method is presented in the Eq. (11).

$$
F(x)=\frac{1}{\pi \lambda\left[1+\left(\frac{x-x_{0}}{\gamma}\right)^{2}\right]}
$$

Where location parameter is denoted as $x_{0}$ and $\gamma$ parameter is should be greater than $0 \gamma>0$. The random variable in the distribution is denoted as $x$, which has the range of $x \in(-\infty,+\infty)$. In this work, the Eagle Strategy based the PSO algorithm is used for local search, whereas the earlier researchers have used the other optimization techniques namely ACO, $\mathrm{ABC}$ and PSO for local search. In addition, the global search is carried out by Levy walks with randomization. In the meta-heuristics algorithms, there are various stopping criteria the number of iterations reaching maximum number, a fixed number of generations, no change in the average fitness after some generations and a located string with a certain value. But, the tolerance values with maximum number of iterations are selected as stopping criteria $(|\operatorname{minf}(k+1)-\min f(k)|<$ tolerance) in this study, which is presented in the Fig. 1.

\section{Experimental design}

Cloud datacenters energy management helps to save more energy and increases the profit of the company. Many optimization methods have been proposed for efficient energy management in the cloud datacenter and these methods has the limitation of more SLA violation. The ES-MPSO method is tested on the CloudSim to evaluate the performance. This section provides a details about the system configuration to test the method and metrics used for the analysis. The parameter metrics used in the work are explained below.
To validate the efficiency of ES-MPSO method with existing techniques, we have used the metrics energy consumption, makespan, memory usage and SLA violations. Energy consumption and SLA violation are already explained in the Eq. (3) and (6), whereas the explanation of makespan is given in the below section.

Makespan: The total time for the system to complete all tasks is defined as makespan. It is also defined as when the request is received from the time to the completion of last task time. The Eq. (12) shows the formula for makespan,

$$
\text { Makespan }=\text { Minimize }\left\{\begin{array}{c}
\max \\
t_{i} \in T_{z}, V_{k} \in V
\end{array} f t_{i, k}\right\}
$$

Where, $f t_{i, k}$ is the finishing time of task $t_{i}$ on the virtual machine $V_{k}$.

System Configuration: The developed ES-MPSO method is evaluated in the system consists of Intel i7 processor with 8 GB of RAM and 500 GB hard disk. The CloudSim tool is used to simulate the cloud datacenter environment. The total number of user requests (i.e. 250 tasks) is used to evaluate the performance of the proposed and existing methods. The proposed and existing methods are simulated in the same environment. The configuration of VM is presented in the Table 1.

\section{Experimental results}

Energy management of the cloud datacenter is a challenging process due to allocation issues of optimal resources. In this research work, the ESMPSO method is proposed for minimizing the SLA violation and energy consumption in the cloud datacenter. The proposed and existing method is simulated in CloudSim to evaluate the performance. The proposed ES-MPSO method is compared with the existing firefly based optimization method [14] and standard PSO method. Various metrics like energy consumption, makespan, memory usage and SLA violation were measured and compared with existing methods.

Table 1. VM configuration

\begin{tabular}{|c|c|}
\hline Parameters & Values \\
\hline Total Number of VM & 8 \\
\hline Processing Speed (MIPS) & 250 \\
\hline RAM (MB) & 512 \\
\hline Bandwidth (Mbps) & 1000 \\
\hline VM manager & Xen \\
\hline Operating System & Linux \\
\hline
\end{tabular}


Table 2. Energy Consumption ( $\mathrm{J}$ ) of the existing and proposed method

\begin{tabular}{|c|c|c|c|c|c|}
\hline $\begin{array}{c}\text { Number } \\
\text { of user requested task }\end{array}$ & $\begin{array}{c}\text { Two- } \\
\text { stage strategy [14] }\end{array}$ & RALBA [14] & $\begin{array}{c}\text { NMT- } \\
\text { FOLS [14] }\end{array}$ & PSO & $\begin{array}{c}\text { Proposed Method } \\
\text { (ES-MPSO) }\end{array}$ \\
\hline 100 & 45 & 43 & 35 & 34 & 28 \\
\hline 125 & 50 & 48 & 38 & 38 & 31 \\
\hline 150 & 59 & 54 & 44 & 41 & 36 \\
\hline 175 & 67 & 58 & 46 & 43 & 38 \\
\hline 200 & 74 & 64 & 50 & 48 & 42 \\
\hline 225 & 79 & 68 & 52 & 49 & 45 \\
\hline 250 & 83 & 73 & 58 & 51 & 48 \\
\hline
\end{tabular}

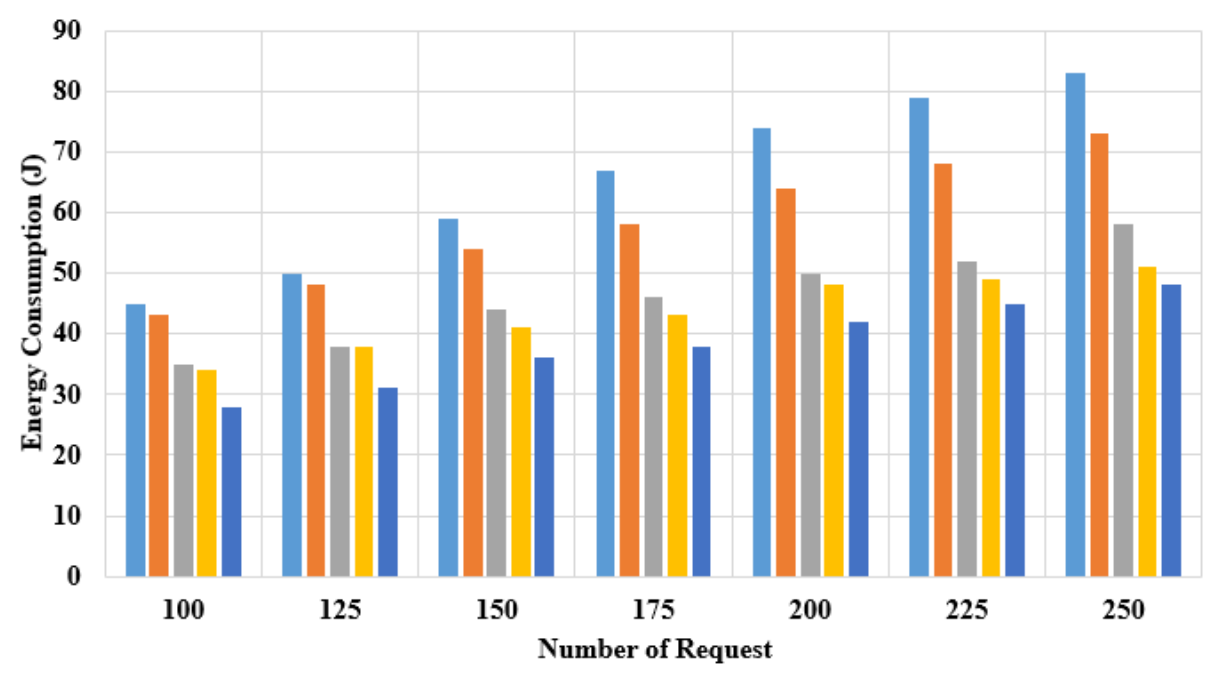

$\approx$ Two-stage strategy $\backsim$ RALBA $\approx$ NMT-FOLS $\approx$ PSO $\backsim$ Proposed Method (ES-MPSO)

Figure. 2 Energy consumption of proposed ES-MPSO and existing method

Table 3. Makespan of the proposed ES-MPSO and existing methods

\begin{tabular}{|c|c|c|c|c|c|}
\hline $\begin{array}{c}\text { Number of user } \\
\text { requested task }\end{array}$ & $\begin{array}{c}\text { Two-stage } \\
\text { strategy [14] }\end{array}$ & RALBA [14] & $\begin{array}{c}\text { NMT-FOLS } \\
{[\mathbf{1 4}]}\end{array}$ & PSO & ES-MPSO \\
\hline 100 & 34 & 29 & 20 & 14 & 11 \\
\hline 125 & 41 & 35 & 24 & 18 & 14 \\
\hline 150 & 48 & 42 & 27 & 24 & 21 \\
\hline 175 & 54 & 46 & 30 & 28 & 24 \\
\hline 200 & 60 & 50 & 32 & 29 & 26 \\
\hline 225 & 65 & 54 & 34 & 32 & 28 \\
\hline 250 & 70 & 58 & 35 & 33 & 29 \\
\hline
\end{tabular}

Table 4. Memory usage of the proposed ES-MPSO and existing methods

\begin{tabular}{|c|c|c|c|c|c|}
\hline $\begin{array}{c}\text { Number of user } \\
\text { requested task }\end{array}$ & $\begin{array}{c}\text { Two-stage strategy } \\
{[\mathbf{1 4}]}\end{array}$ & $\begin{array}{c}\text { RALBA } \\
{[\mathbf{1 4}]}\end{array}$ & $\begin{array}{c}\text { NMT-FOLS } \\
{[\mathbf{1 4}]}\end{array}$ & PSO & $\begin{array}{c}\text { Proposed Method (ES- } \\
\text { MPSO) }\end{array}$ \\
\hline 100 & 49 & 52 & 29 & 26 & 21 \\
\hline 125 & 55 & 59 & 33 & 28 & 23 \\
\hline 150 & 69 & 66 & 36 & 31 & 28 \\
\hline 175 & 81 & 74 & 41 & 37 & 32 \\
\hline 200 & 93 & 86 & 52 & 49 & 45 \\
\hline 225 & 111 & 94 & 61 & 53 & 61 \\
\hline 250 & 122 & 105 & 72 & 68 & 69 \\
\hline
\end{tabular}

The Table 2 presents comparison between ESMPSO method and the existing methods based on the energy consumption. The simulation result shows that the ES-MPSO method has lower energy consumption compared to other existing methods. The ES-MPSO method is compared with the standard PSO method based on the energy management in the cloud datacenter. This shows that the ES-MPSO 
method has lower energy consumption compared to standard PSO and existing method. The ES-MPSO method is able to achieve better convergence performance due to Cauchy mutation process. The ES-MPSO method has the energy consumption of 38 $\mathrm{J}$ while the existing method NMT-FOLS consumed energy of $46 \mathrm{~J}$.

The Fig. 2 presents graphical comparison between ES-MPSO and existing methods based on the energy consumption. The result indicates that ESMPSO method has lower energy consumption compared to existing method in terms of load balancing of various requests. The ES-MPSO method has achieved lower energy consumption compared to standard PSO and existing NMT-FOLS method. The ES-MPSO method consumed energy of $42 \mathrm{~J}$ for 200 number of requests, while existing NMT-FOLS method has consumed $50 \mathrm{~J}$ energy.

The Fig. 3 presents the makespan of the ESMPSO and existing methods. The makespan is measured for a number of user request from the range of 100 to 250 . The simulation result shows that proposed ES-MPSO method achieves lower makespan for the various numbers of user request. The proposed ES-MPSO method has lower makespan due to its searching process of Eagle Strategy. The proposed ES-MPSO method has achieved makespan of $24 \mathrm{~ms}$ for 175 number of the user request and the existing method NMT-FOLS has achieved $30 \mathrm{~ms}$ of makespan.

The Table 3 presents makespan of The ES-MPSO method and existing method makespan. The proposed ES-MPSO method has lower makespan compared to the existing method. Lower makespan is achieved by the proposed ES-MPSO compared to standard PSO and existing methods in the energy management of cloud datacentre. Makespan of ESMPSO method is $21 \mathrm{~ms}$ for 150 requests, while the existing method PSO has 24 ms makespan.

The Fig. 4 presents the memory usage of the proposed ES-MPSO and existing firefly based method. The simulation results of the memory usage indicate that memory usage of ES-MPSO method is lower than the existing method. The existing method requires more data for the optimization, whereas the ES-MPSO method requires less amount of data in the searching process. Therefore, the proposed ESMPSO method requires lower memory usage compared to the existing method NMT-FOLS. The ES-MPSO method has achieved the memory usage of $28 \mathrm{MB}$, while the NMT-FOLS has used $36 \mathrm{MB}$ of memory.

The Table 4 shows memory usage of the ESMPSO and existing methods. The proposed ESMPSO method has lower memory requirements compared to the existing method. The ES-MPSO method requires $28 \mathrm{MB}$ of memory for 150 requests and existing method NMT-FOLS requires $36 \mathrm{MB}$ memory.

The Fig. 5 presents average SLA violation of the proposed ES-MPSO and the PSO method. The SLA violation is measured in term of the number of CPU and memory provided to the user. The proposed ESMPSO method achieves lower SLA violation compared to the PSO method, because The ESMPSO method has better convergence property that improve search process. The SLA violation of the ES-MPSO method is 12 and PSO is 14.04 SLA violation for 250 user requests.

The efficiency of the ES-MPSO method is high

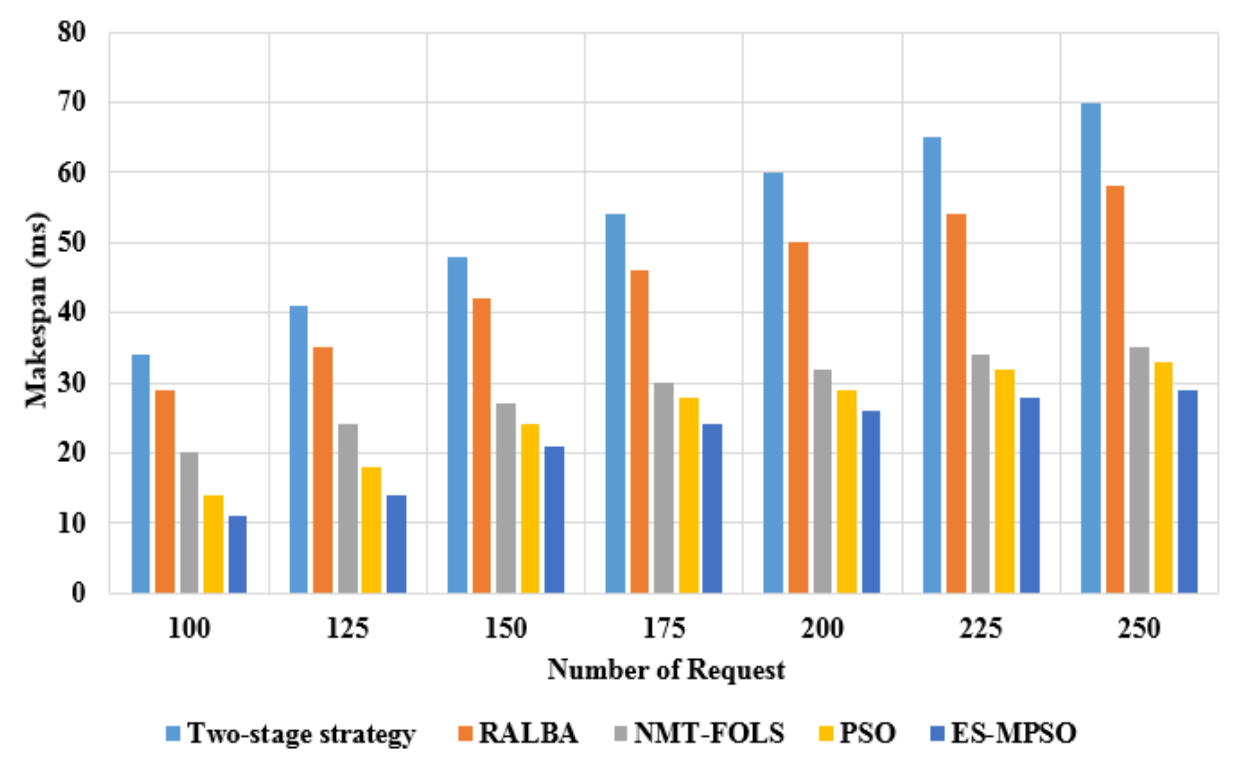

Figure. 3 Makespan of the ES-MPSO method and other existing methods 


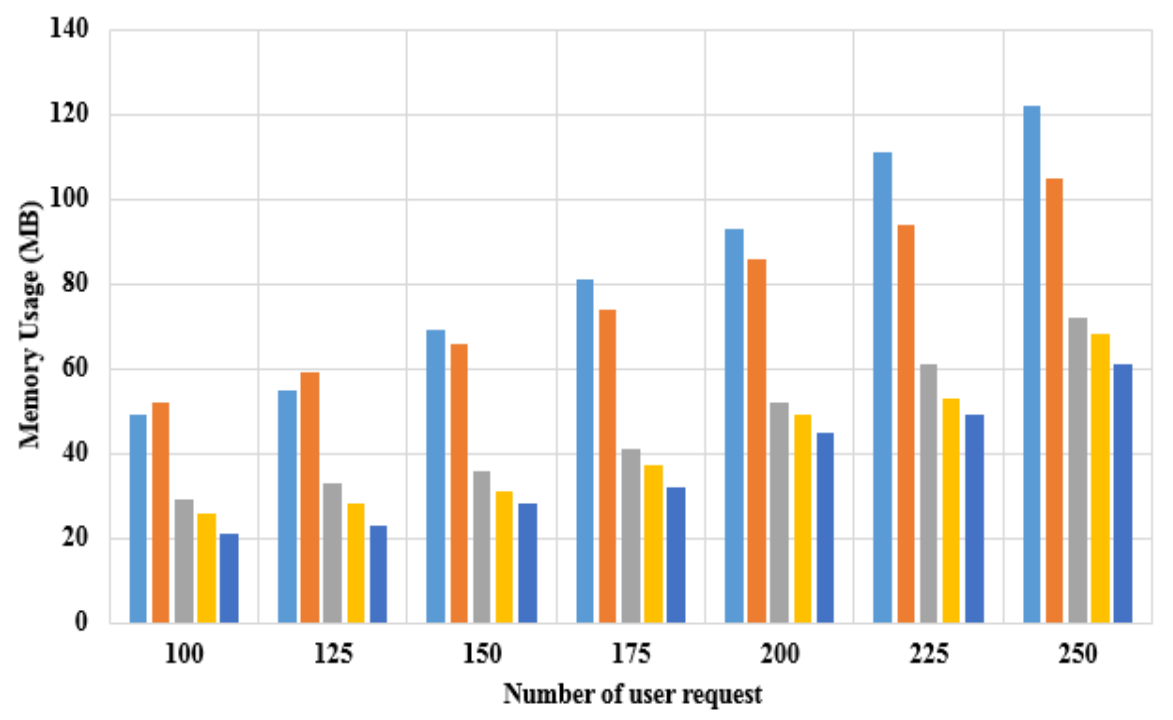

$\backsim$ Two-stage strategy $\backsim$ RALBA $\approx$ NMT-FOLS $\backsim$ PSO $\backsim$ Proposed Method (ES-MPSO)

Figure. 4 The memory usage of the proposed method and other existing method

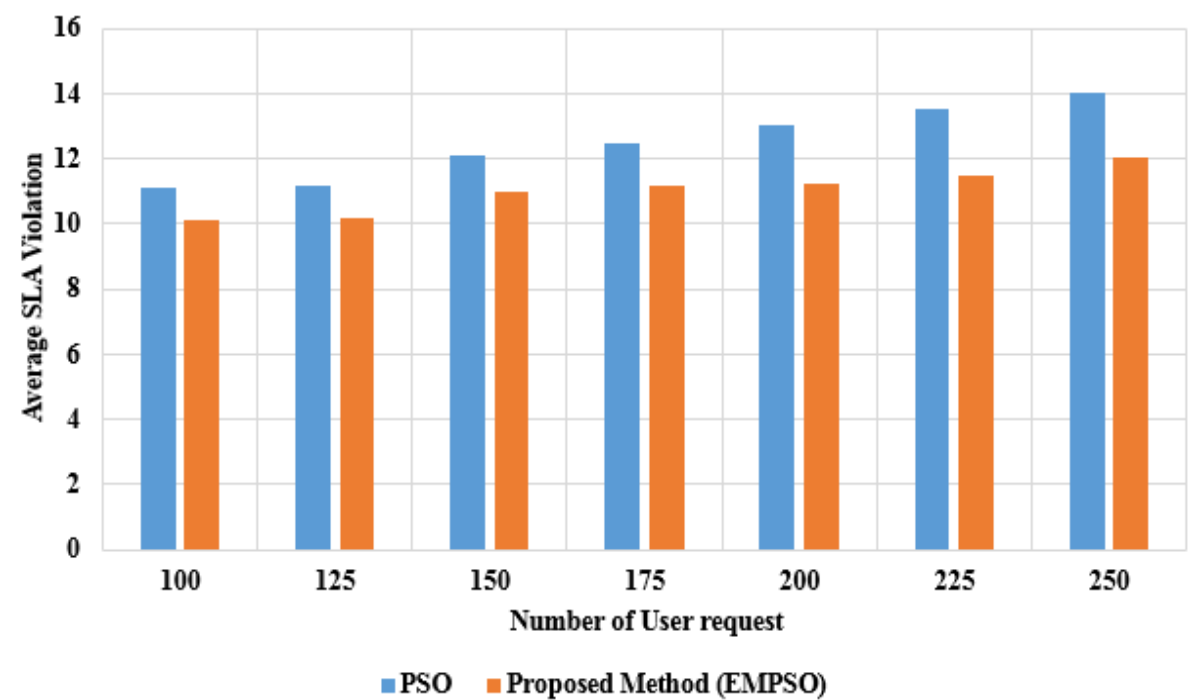

Figure. 5 The SLA violation of ES-MPSO and PSO method

compared to the PSO method for the energy management in the cloud datacenter. In addition, the effectiveness of proposed method is studied with case study that is described next.

In the case study, the proposed MPSO is altered with another algorithm called ACO to test the effectiveness of proposed ES. Hence, the proposed method is defined as ES-ACO. The simulation of proposed ES-ACO method is conducted on other existing techniques MACO [13], H_BAC, ABC, $\mathrm{ACO}$ and OH_BAC [17] in terms of energy consumption and SLA violations. Table 5 shows the comparative analysis of the proposed method based on the SLA violations with a number of user requested tasks. From the Table 5, it is clearly stated that the proposed ES-ACO has less SLA violation compare to other existing techniques. For instance, ES-ACO has only 1.2 SLA violation, where ABC and H_BAC has nearly 4.8 to 5.0 SLA violation and OH_BAC has 1.8 SLA violation.

The reason is that the ES is applied to increase the ACO algorithm by Levy distribution, where the other techniques are not presented with ES. This case study proves that the effectiveness of meta-heuristic algorithms are greatly improved by the ES. The Table 6 shows the comparative analysis of case study in terms of energy consumption.

When the user requested tasks increase, the energy consumption of proposed ES-ACO also increase. For instance, it achieved only 12J, when the task is 50 and it achieved 62J, when the task is 250 . However, the performance of the ES-ACO provides 
Table 5. Comparative analysis of proposed ES-ACO in terms of SLA violation

\begin{tabular}{|c|c|c|c|c|c|c|}
\hline $\begin{array}{c}\text { No. of user } \\
\text { requested task }\end{array}$ & MACO [13] & ACO [17] & ABC [17] & H_BAC [17] & $\begin{array}{c}\text { OH_BAC } \\
{[17]}\end{array}$ & $\begin{array}{c}\text { Proposed ES- } \\
\text { ACO }\end{array}$ \\
\hline 50 & 1.5 & 4.9 & 4.0 & 3.0 & 2.0 & 1.3 \\
\hline 100 & 1.3 & 11.0 & 4.8 & 5.0 & 1.8 & 1.2 \\
\hline 150 & 1.05 & 15.0 & 5.0 & 10.0 & 1.5 & 1.0 \\
\hline 200 & 1.03 & 22.0 & 3.0 & 13.0 & 1.0 & 0.9 \\
\hline 250 & 1.0 & 30.0 & 2.0 & 13.0 & 0.5 & 0.5 \\
\hline
\end{tabular}

Table 6. Comparative analysis of proposed ES-ACO by means of energy consumption (J)

\begin{tabular}{|c|c|c|c|c|c|c|}
\hline $\begin{array}{c}\text { No. of user } \\
\text { requested task }\end{array}$ & $\begin{array}{c}\text { MACO } \\
{[13]}\end{array}$ & ACO [17] & ABC [17] & H_BAC [17] & $\begin{array}{c}\text { OH_BAC } \\
{[17]}\end{array}$ & $\begin{array}{c}\text { Proposed ES- } \\
\text { ACO }\end{array}$ \\
\hline 50 & 16 & 33 & 30 & 49 & 18 & 12 \\
\hline 100 & 35 & 52 & 50 & 59 & 38 & 28 \\
\hline 150 & 48 & 68 & 68 & 71 & 55 & 36 \\
\hline 200 & 64 & 79 & 82 & 90 & 70 & 49 \\
\hline 250 & 78 & 82 & 95 & 99 & 82 & 62 \\
\hline
\end{tabular}

better performance than other existing techniques. The traditional ACO achieved 68J, MACO achieved 48J and OH_BAC achieved 55J for the task 150, where the proposed ES-ACO achieved only 36J for the same task. The reason is that both the local and global search are performed by the ES effectively, which assigns the tasks and minimized the energy consumption effectively. Therefore, the case study proves that the proposed method achieved better performance compared to the other traditional techniques and hybrid meta-heuristics algorithms.

\section{Conclusion}

Energy management in cloud datacenter is an essential process and optimized resource allocation is required to that increase the profits. In recent years, several optimization methods have been developed for the efficient energy management in the cloud datacenter. The proposed ES-MPSO method improves efficiency in energy management at cloud datacenters. Rigorous local search is performed by the PSO technique and the Cauchy Mutation improved the performance of convergence in the PSO. The simulation results showed that the ES-MPSO method gained higher efficiency in the energy management and reduced the SLA violations. The ES-MPSO method had consumed energy of 48J for 250 user requests, while the NMT-FOLS and PSO had consumed $58 \mathrm{~J}$ and $51 \mathrm{~J}$ of energy. In the future work, the multi-objective optimization method will be proposed and implemented to further increase the energy efficiency in cloud datacenters.

\section{Conflicts of Interest}

The authors declare no conflict of interest.

\section{Author contributions}

The first author of the paper contributed in conceptualization, methodology, software validation and verification, formal analysis, investigation, resources, data curation, writing original draft, writing review and editing, visualization. The supervision and management of the project was carried out by the second author.

\section{References}

[1] G. S. Aujla and N. Kumar, "MEnSuS: An efficient scheme for energy management with sustainability of cloud data centers in edge-cloud environment", Future Generation Computer Systems, Vol. 86, pp. 1279-1300, 2018.

[2] E. Arianyan, H. Taheri, and V. Khoshdel, "Novel fuzzy multi objective DVFS-aware consolidation heuristics for energy and SLA efficient resource management in cloud data centers", Journal of Network and Computer Applications, Vol. 78, pp. 43-61, 2017.

[3] M. Vasudevan, Y. C. Tian, M. Tang, E. Kozan and X. Zhang, "Energy-efficient application assignment in profile-based data center management through a Repairing Genetic Algorithm", Applied Soft Computing, Vol. 67, pp. 399-408, 2018.

[4] S. L. Chen, Y. Y. Chen, and S.H. Kuo, "CLB: A novel load balancing architecture and algorithm for cloud services", Computers \& Electrical Engineering, Vol. 58, pp. 154-160, 2017.

[5] K. Muralitharan, R. Sakthivel, and Y. Shi, "Multiobjective optimization technique for demand side management with load balancing approach in smart grid", Neurocomputing, Vol. 177, pp. 110-119, 2016. 
[6] M. Lawanyashri, B. Balusamy, and S. Subha, "Energy-aware hybrid fruitfly optimization for load balancing in cloud environments for EHR applications", Informatics in Medicine Unlocked, Vol. 8, pp. 42-50, 2017.

[7] V. Simic, B. Stojanovic, and M. Ivanovic, "Optimizing the performance of optimization in the cloud environment-An intelligent autoscaling approach", Future Generation Computer Systems, Vol. 101, pp. 909-920, 2019.

[8] D. Kesavaraja and A. Shenbagavalli, "QoE enhancement in cloud virtual machine allocation using Eagle strategy of hybrid krill herd optimization", Journal of Parallel and Distributed Computing, Vol. 118, pp. 267-279, 2018.

[9] T. P. Shabeera, S. M. Kumar, S. M. Salam, and K.M. Krishnan, "Optimizing VM allocation and data placement for data-intensive applications in cloud using ACO metaheuristic algorithm. Engineering Science and Technology", An International Journal, Vol. 20, No. 2, pp. 616628, 2017.

[10] F. Sheikholeslami and N. J. Navimipour, "Service allocation in the cloud environments using multi-objective particle swarm optimization algorithm based on crowding distance", Swarm and Evolutionary Computation, Vol. 35, pp. 53-64, 2017.

[11] K. M. Cho, P. W. Tsai, C. W. Tsai, and C. S. Yang, "A hybrid meta-heuristic algorithm for VM scheduling with load balancing in cloud computing", Neural Computing and Applications, Vol. 26, No. 6, pp. 1297-1309, 2015.

[12] Z. Zhou, J. Abawajy, M. Chowdhury, Z. Hu, K. $\mathrm{Li}$, H. Cheng, A. A. Alelaiwi, and F. Li, "Minimizing SLA violation and power consumption in Cloud data centers using adaptive energy-aware algorithms", Future Generation Computer Systems, Vol. 86, pp. 836-850, 2018.

[13] M. H. Malekloo, N. Kara, and M. El Barachi, "An energy efficient and SLA compliant approach for resource allocation and consolidation in cloud computing environments", Sustainable Computing: Informatics and Systems, Vol. 17, pp. 9-24, 2018.

[14] J. Prassanna and N. Venkataraman, "Adaptive regressive holt-winters workload prediction and firefly optimized lottery scheduling for load balancing in cloud", Wireless Networks, pp. 1-19, 2019.

[15] T. Jena and V. Mohanty, "GA-based customerconscious resource allocation and task scheduling in multi-cloud computing", Arabian
Journal for Science and Engineering, Vol. 43, No. 8, pp. 4115-4130, 2018.

[16] L. Li, J. Dong, D. Zuo, and J. Wu, "Sla-aware and energy-efficient VM consolidation in cloud data centers using robust linear regression prediction model", IEEE Access, Vol. 7, pp. 9490-9500, 2019.

[17] M. Gamal, R. Rizk, H. Mahdi, and B. E. Elnaghi, "Osmotic Bio-Inspired Load Balancing Algorithm in Cloud Computing", IEEE Access, Vol. 7, pp. 42735-42744, 2019.

[18] H. Yapıc1 and N. Çetinkaya, "An improved particle swarm optimization algorithm using eagle strategy for power loss minimization", Mathematical Problems in Engineering, Vol. 2017, 2017.

[19] A. Sarangi, S. Samal, and S.K. Sarangi, "Analysis of Gaussian \& Cauchy Mutations in Modified Particle Swarm Optimization Algorithm", In: Proc. of the $5^{\text {th }}$ International Conf. on Advanced Computing \& Communication Systems (ICACCS), pp. 463-467, 2019. 